\title{
Further results on Maximal Nontraceable graphs of smallest size
}

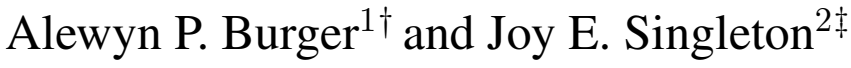 \\ ${ }^{1}$ Department of Logistics, Stellenbosch University \\ ${ }^{2}$ Department of Mathematical Sciences, University of South Africa \\ received $16^{\text {th }}$ April 2012, revised $28^{\text {th }}$ January 2013, accepted $28^{\text {th }}$ January 2013.
}

Let $g(n)$ denote the minimum number of edges of a maximal nontraceable (MNT) graph of order $n$. In 2005 Frick and Singleton (Lower bound for the size of maximal nontraceable graphs, Electronic Journal of Combinatorics, 12(1) R32, 2005) proved that $g(n)=\left\lceil\frac{3 n-2}{2}\right\rceil$ for $n \geq 54$ as well as for $n \in I$, where $I=\{12,13,22,23,30,31,38,39$, $40,41,42,43,46,47,48,49,50,51\}$ and they determined $g(n)$ for $n \leq 9$. We determine $g(n)$ for 18 of the remaining 26 values of $n$, showing that $g(n)=\left\lceil\frac{3 n-2}{2}\right\rceil$ for $n \geq 54$ as well as for $n \in I \cup\{18,19,20,21,24,25,26,27,28$, $29,32,33\}$ and $g(n)=\left\lceil\frac{3 n}{2}\right\rceil$ for $n \in\{10,11,14,15,16,17\}$. We give results based on "analytic" proofs as well as computer searches.

Keywords: maximal nontraceable, $x y$-maximal nontraceable, $z$-maximal nontraceable, maximal nonhamiltonian

\section{Introduction}

We consider only simple, finite graphs $G$ and denote the vertex set, the edge set and the size (number of edges) of $G$ by $V(G), E(G)$ and $e(G)$, respectively. We denote the complement of $G$ by $\bar{G}$. The degree of a vertex $v$ in a graph $G$ is denoted by $d_{G}(v)$. If no confusion can result we simply write $d(v)$. A vertex $v \in V(G)$ is a universal vertex of $G$ if it is adjacent to all vertices in $G-v$. The open neighbourhood of a vertex $v$ in $G$ is the set $N_{G}(v)=\{x \in V(G): v x \in E(G)\}$. The open neighbourhood of a subgraph $H$ of $G$ is the set $N_{G}(H)=\{x \in V(G)-V(H): v x \in E(G)$ for at least one $v \in V(H)\}$. If $S$ is a nonempty subset of $V(G)$, then $\langle S\rangle$ denotes the subgraph of $G$ induced by $S$.

A graph $G$ is hamiltonian if it has a Hamilton cycle (a cycle containing all the vertices of $G$ ), and traceable if it has a Hamilton path (a path containing all the vertices of $G$ ). A graph $G$ is maximal nonhamiltonian (MNH) if $G$ is not hamiltonian, but $G+e$ is hamiltonian for each $e \in E(\bar{G})$. A graph $G$ is maximal nontraceable (MNT) if $G$ is not traceable, but $G+e$ is traceable for each $e \in E(\bar{G})$. Let $x$ and $y$ be distinct vertices in $G$. Then $G$ is $x y$-traceable if $G$ is traceable between $x$ and $y$, i.e. has a

\footnotetext{
${ }^{\dagger}$ Email: apburgeresun.ac.za. Research towards this paper was supported financially by the South African National Research Foundation under grant number GUN 77248.

‡Email: singlje@unisa.ac.za.

1365-8050 @ 2013 Discrete Mathematics and Theoretical Computer Science (DMTCS), Nancy, France
} 
Hamilton path with $x$ and $y$ as endvertices. Also, $G$ is $x y$-maximal nontraceable ( $x y-\mathrm{MNT}$ ) if $G$ is not $x y$-traceable ( $x y$-nontraceable), but $G+e$ is $x y$-traceable for each $e \in E(\bar{G})$. A graph $G$ is $z$-traceable if it is traceable from $z$ (i.e. there exists a Hamilton path with $z$ as an endvertex), and it is $z$-maximal nontraceable ( $z$-MNT) if $G$ is not $z$-traceable, but $G+e$ is $z$-traceable for each $e \in E(\bar{G})$. A graph $G$ is hypohamiltonian if it is not hamiltonian, but $G-v$ is hamiltonian for every $v \in V(G)$.

In [1] Bollobás posed the problem of finding the least number of edges in an $\mathrm{MNH}$ graph of given order. This problem was solved by combined results given in [2, 5, 6, 9]. A similar problem was investigated for MNT graphs (also referred to as hamiltonian path saturated (HPS) graphs) as well as for $m$-path cover saturated $(m$ PCS) graphs, which are generalizations of HPS graphs, in [7, 8, 12, 13]. Suppose $g(n)$ denotes the minimum number of edges of an MNT graph of order $n$. Frick and Singleton [8] showed that $g(n)=$ $\left\lceil\frac{3 n-2}{2}\right\rceil$ for $n \geq 54$ as well as for $n \in I$, where $I=\{12,13,22,23,30,31,38,39,40,41,42,43,46,47,48$, $49,50,51\}$ and determined $g(n)$ for $n \leq 9$. In this paper we show that $g(n)=\left\lceil\frac{3 n}{2}\right\rceil$ for $n=10,11,14,15$, 16,17 and $g(n)=\left\lceil\frac{3 n-2}{2}\right\rceil$ for $n \in\{18,19,20,21,24,25,26,27,28,29,32,33\}$ and supply graphs having the minimum number of edges.

Many MNT graphs have either $x y-\mathrm{MNT}$ graphs or $z$-MNT graphs as subgraphs and thus we have a section devoted to $x y$-MNT graphs and $z-$ MNT graphs of smallest size.

\section{Preliminary results}

For easy reference we include certain results from [8].

Lemma 2.1 [8] If $G$ is a connected MNT graph and $v \in V(G)$ with $d(v)=2$, then the neighbours of $v$ are adjacent. Also, one of the neighbours has degree at least 4 and the other neighbour has degree 2 or at least 4 .

Theorem 2.2 [8] Suppose $G$ is a connected MNT graph without vertices of degree 1 or adjacent vertices of degree 2. If $G$ has order $n \geq 7$ and $m$ vertices of degree 2 , then $e(G) \geq \frac{1}{2}(3 n+m)$.

We include the main steps of the proof of the following theorem, as the proofs of our main results relate to the construction and cases used in this proof.

Theorem 2.3 [8] If $G$ is an MNT graph of order n, then

$$
e(G) \geq \frac{3 n-2}{2} \text { for } n \geq 10
$$

Proof: If $G$ is not connected, then $G=K_{k} \cup K_{n-k}$, for some positive integer $k<n$ and then, clearly, $e(G)>\frac{3 n-2}{2}$ for $n \geq 10$. Thus we assume that $G$ is connected.

We need to prove that the sum of the degrees of the vertices of $G$ is at least $3 n-2$. In view of Theorem 2.2, we let

$$
M=\{v \in V(G) \mid d(v)=2 \text { and no neighbour of } v \text { has degree } 2\} .
$$

The remaining vertices of degree 2 can be dealt with simultaneously with the vertices of degree 1 . We let

$$
S=\{v \in V(G)-M \mid d(v)=2 \text { or } d(v)=1\} .
$$


Let $m=|M|$ and $s=|S|$. If $S=\emptyset$, then it follows from Theorem 2.2 that $e(G) \geq \frac{1}{2}(3 n+m)$. Thus we assume $S \neq \emptyset$.

We observe that, if $H$ is a component of the graph $\langle S\rangle$, then either $H \cong K_{1}$ or $H \cong K_{2}$ and $N_{G}(H)$ consists of a single vertex, which is a cut-vertex of $G$.

An example of such a graph $G$ is depicted in Figure 1

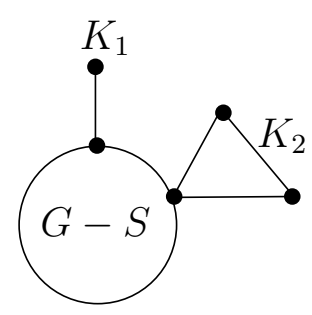

Fig. 1: Example of $G$

Since $G$ is MNT the graph $\langle S\rangle$ has at most three components. We consider three cases:

Case 1. $\langle S\rangle$ has exactly three components, say $H_{1}, H_{2}, H_{3}$ :

In this case the neighbourhoods of $H_{1}, H_{2}, H_{3}$ are pairwise disjoint, $G-S$ is a complete graph of order at least 4 and hence

$$
e(G)=\left(\begin{array}{c}
n-s \\
2
\end{array}\right)+2 s-3 \text { for } s=3,4,5 \text { or } 6 .
$$

This case is a Zelinka Type II construction, cf. [14].

Case 2. $\langle S\rangle$ has exactly two components, say $H_{1}, H_{2}$ :

There are two subcases.

Case 2.1. $N_{G}\left(H_{1}\right)=N_{G}\left(H_{2}\right)$ :

Then $G-S$ is a complete graph and hence

$$
e(G)=\left(\begin{array}{c}
n-s \\
2
\end{array}\right)+2 s-2 \text { for } s=2,3 \text { or } 4 .
$$

This case is a Zelinka Type I construction, cf. [14].

Case 2.2. $N_{G}\left(H_{1}\right) \neq N_{G}\left(H_{2}\right)$ :

Let $N_{G}\left(H_{i}\right)=y_{i}, i=1,2$ and $y_{1} \neq y_{2}$. Then $y_{1} y_{2} \in E(G)$.

Now $G-S$ is not complete. If $v$ and $w$ are nonadjacent vertices in $V(G-S)$, then $(G-S)+v w$ has a Hamilton cycle containing $y_{1} y_{2}$. Hence $G-S$ is either hamiltonian or MNH. We consider these two cases separately:

Case 2.2.1. $G-S$ is hamiltonian:

We note that no Hamilton cycle in $G-S$ contains $y_{1} y_{2}$, otherwise $G$ would be traceable. Also $d_{G-S}\left(y_{i}\right) \geq 3$ for $i=1,2$. We have

$$
e(G)=e(G-M-S)+2 m+2 s-2 \geq \frac{1}{2}(3 n+m+s-4) .
$$

Case 2.2.2. $G-S$ is nonhamiltonian, i.e. $G-S$ is $\mathrm{MNH}$ : 
We have

$$
e(G)=e(G-S)+2 s-2 \geq \frac{1}{2}(3 n+s-4) .
$$

Case 3. $\langle S\rangle$ has exactly one component, say $H$ :

Since

$$
\sum_{v \in S} d_{G}(v)=3 s-2, \text { for } s=1,2
$$

it follows that

$$
\begin{aligned}
e(G) & =e(G-M)+2 m \\
& =\frac{1}{2}\left(\sum_{v \in V(G-M)-S} d_{G-M}(v)+\sum_{v \in S} d_{G-M}(v)\right)+2 m \\
& \geq \frac{1}{2}(3(n-m-s)+3 s-2)+2 m .
\end{aligned}
$$

Hence

$$
e(G) \geq \frac{1}{2}(3 n+m-2) .
$$

Remark 2.4 We note the following:

(a) If $G$ is an MNT graph as described in Case 1 of Theorem 2.3, then

$$
e(G)>\frac{3 n}{2} \text { if } n>10 .
$$

(b) If $G$ is an MNT graph as described in Case 2.1 of Theorem 2.3. then

$$
e(G)>\frac{3 n}{2} \text { if } n>8 .
$$

(c) The subgraphs $G-S$ in Cases 2.2.1 and 2.2.2 are $y_{1} y_{2}-M N T$ graphs. The subgraph $G-S$ in Case 3 is a $z-M N T$ graph, where $z=N_{G}(H)$.

We introduce the following terminology which we require in proving certain results in order to show that $g(10)=15$.

A vertex $v$ in a subgraph $H$ of a graph $G$ is an attachment vertex of $H$ if $v$ has a neighbour in $V(G)-$ $V(H)$. The circumference $c(G)$ of a graph $G$ is the order of a longest cycle in $G$. We suppose that a cycle $C$ has an orientation. If $u, v \in V(C)$ we denote the path on $C$ from $u$ to $v$ (with same orientation as $C$ ) by $C[u, v]$ and the other path on $C$ from $u$ to $v$ by $\bar{C}[u, v]$. The paths obtained by deleting the endvertices $u, v$ are denoted by $C(u, v)$ and $\bar{C}(u, v)$, respectively.

Lemma 2.5 Suppose $C$ is a circumference cycle of a graph $G$ and $G-V(C)$ has a path $P=y L z$ with endvertices $y$ and $z$, where we also consider $V(L)=\emptyset$ as well as $y=z$. If $y$ is adjacent to $u \in V(C)$ and $z$ is adjacent to $v \in V(C), u \neq v$, then

$$
|V(C(u, v))| \geq|V(P)| \text { and }|V(\bar{C}(u, v))| \geq|V(P)| .
$$


Proof: Suppose $|V(C(u, v))|<|V(P)|$. Then $C^{\prime}=\bar{C}[u, v] z L y u$ is a cycle such that $\left|V\left(C^{\prime}\right)\right|>|V(C)|$ which is a contradiction since $C$ is a circumference cycle. A similar argument holds if $|V(\bar{C}(u, v))|<$ $|V(P)|$.

The following lemma is a direct result of Lemma 2.5

Lemma 2.6 Suppose $c(G)=k$. Let $C$ be a circumference cycle of $G$. Suppose $u, v \in V(G-V(C))$ are endvertices of a path in $G-V(C)$ of order at least $\left\lfloor\frac{k}{2}\right\rfloor$. If both $u$ and $v$ are attachment vertices of $G-V(C)$, then $\left|N_{C}(\{u, v\})\right|=1$.

We also require the following lemma in order to prove $g(10)=15$.

Lemma 2.7 Suppose $G$ is a graph of order 10 as described in Case 3 of Theorem 2.3. with $H \cong K_{1}$ and $d_{G}(v)=3$ for all $v \in V(G)-S$. Then $G-S$ is 2-connected.

Proof: Suppose $G-S$ has a cut vertex $y$ and that $G$ has the structure as depicted in Figure 2 .

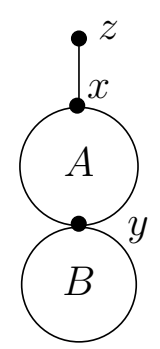

Fig. 2: Sketch for Lemma 2.7

Consider $G+z y$. Since $G+z y$ is traceable it follows that $B$ is traceable from $y$. If $B$ is not complete, then $A$ is traceable between $x$ and $y$ making $G$ traceable. Hence $B$ is complete.

If $B=K_{3}$, then $G$ has two vertices of degree 2, and if $B=K_{4}$, then $d_{G}(y)>3$, both of which lead to contradictions.

Hence $G-S$ is 2 -connected.

\section{$3 \quad x y-$ MNT and $z-$ MNT graphs}

In this section we investigate some properties of $x y-\mathrm{MNT}$ and $z$-MNT graphs and show how these graphs can be used in constructing MNT graphs. We also present some $x y$-MNT graphs of small order and smallest size, as well as some cubic $x y$-MNT graphs of larger orders.

Lemma 3.1 Suppose F is an $x y-M N T$ graph.

(a) Then $x y \in E(F)$.

(b) If $x$ is not a universal vertex of $F$, then $F-x$ is traceable from $y$.

(c) If $N(x) \cap N(y)=\emptyset$, then $F-\{x, y\}$ is traceable from every vertex in $V(F)-\{x, y\}$. 


\section{Proof:}

(a) If $x y \notin E(F)$, then $F+x y$ is not $x y$-traceable.

(b) Suppose $z \notin N(x)$. Then $F+x z$ has a Hamilton path $x z Q y$ and consequently $F-x$ has a Hamilton path $z Q y$. Hence $F-x$ is traceable from $y$.

(c) Let $u \in V(F)-\{x, y\}$. Since $N(x) \cap N(y)=\emptyset$ it follows that $u x \notin E(F)$ or $u y \notin E(F)$. Suppose $u x \notin E(F)$. Then $F+u x$ has a Hamilton path $x u P y$, and hence $u P$ is a Hamilton path of $F-\{x, y\}$, i. e. $F-\{x, y\}$ is traceable from $u$. A similar proof holds if $u y \notin E(F)$.

Lemma 3.2 Suppose $F$ is an $x y-M N T$ graph with $n \geq 6$, where neither $x$ nor $y$ is a universal vertex and $w \in V(F)-\{x, y\}$ with $d(w)=2$.

(a) Then $w$ is nonadjacent to $x$ and $y$.

(b) If $N(w)=\left\{v_{1}, v_{2}\right\}$ and $d\left(v_{i}\right)=4$ for $i=1,2$, then $\left\langle v_{1}, v_{2}, x, y\right\rangle$ is not complete.

\section{Proof:}

(a) Suppose $w$ is adjacent to $x$ and $u \notin N(x)$. Then $F+x u$ is not $x y$-traceable, a contradiction. A similar argument holds if $w$ is adjacent to $y$.

(b) Suppose $d\left(v_{i}\right)=4$ for $i=1,2$ and $\left\langle v_{1}, v_{2}, x, y\right\rangle$ is complete. Then $F+x w$ is not $x y$-traceable, a contradiction.

Lemma 3.3 Suppose $F$ is a $z-M N T$ graph with $n \geq 5$, where $z$ is not a universal vertex and $w \in$ $V(F)-\{z\}$ with $d(w)=2$.

(a) Then $w$ is nonadjacent to $z$.

(b) If $N(w)=\left\{v_{1}, v_{2}\right\}$ and $d\left(v_{i}\right)=4$ for $i=1,2$, then $\left\langle v_{1}, v_{2}, z\right\rangle$ is not complete.

\section{Proof:}

(a) Suppose $w$ is adjacent to $z$ and $v$, and $u \notin N(z)$. Then $F+z u$ has a Hamilton path of the form $z u P v w$. But then $u P v w z$ is a Hamilton path in $F$, a contradiction.

(b) Suppose $d\left(v_{i}\right)=4$ for $i=1,2$ and $\left\langle v_{1}, v_{2}, z\right\rangle$ is complete. Then $F+z w$ has a Hamilton path of the form $z w v_{i} v_{j} P$ or $z w v_{i} Q v_{j}$, where $\{i, j\}=\{1,2\}$. But then $z v_{i} w v_{j} P$ or $z v_{i} Q v_{j} w$ is a Hamilton path in $F$, a contradiction. 
We consider the following construction.

Construction 1: Suppose $F$ is an $x y-\mathrm{MNT}$ graph and $H_{x} \cong K_{n}$ and $H_{y} \cong K_{m}$, for positive integers $n$ and $m$. Suppose the vertex sets $V(F), V\left(H_{x}\right)$ and $V\left(H_{y}\right)$ are disjoint. Then $\left[F, H_{x}, H_{y}\right]$ denotes the graph obtained by joining each vertex of $H_{x}$ to $x$ and each vertex of $H_{y}$ to $y$.

Theorem 3.4 Suppose $F$ is an $x y-M N T$ graph and neither $x$ nor $y$ is a universal vertex of $F$. If $N_{F}(x) \cap$ $N_{F}(y)=\emptyset$, then $G=\left[F, H_{x}, H_{y}\right]$ is $M N T$.

Proof: Since $F$ is not $x y$-traceable it follows that $G$ is not traceable. We now prove that $G+u v$ is traceable for all $u, v \in V(G)$, where $u v \notin E(G)$.

Suppose that $u, v \in V(F)$. Since $F+u v$ has a Hamilton path between $x$ and $y$ it follows that $G+u v$ is traceable.

Suppose $u \in V\left(H_{x}\right) \cup\{x\}$ and $v \in V\left(H_{y}\right)$. Then, since $x$ is not a universal vertex it follows from Lemma 3.1 (b) that there is a Hamilton path in $G+u v$.

Suppose $u \in V\left(H_{x}\right)$ and $v \in V(F)-\{x, y\}$. Then, from Lemma 3.1. (c), there is a Hamilton path in $G+u v$.

All other cases for $u$ and $v$ follow from symmetry.

If $G=\left[F, H_{x}, H_{y}\right]$, where $F$ is $x y$-MNT and $N_{F}(x) \cap N_{F}(y) \neq \emptyset$, then in order to show that $G$ is MNT one needs to check if $G+u v$ is traceable for (i) $u \in H_{x}$ and $v \in H_{y}$, (ii) $u \in H_{x}$ and $v=y$, (iii) $u=x$ and $v \in H_{y}$, and (iv) $u \in H_{x} \cup H_{y}$ and $v \in N(x) \cap N(y)$.

We now consider $z$-MNT graphs. It follows from the definition that if $F^{z}$ is a $z-\mathrm{MNT}$ graph, then $F^{z}-z$ is traceable from each $v \notin N_{F z}(z)$, but not traceable from any $u \in N_{F z}(z)$.

Construction 2: Suppose $F^{z}$ is a $z-\mathrm{MNT}$ graph and $H_{z} \cong K_{n}$. Suppose the vertex sets $V\left(F^{z}\right)$ and $V\left(H_{z}\right)$ are disjoint. Then $\left[F^{z}, H_{z}\right]$ denotes the graph obtained by joining each vertex of $H_{z}$ to $z$.

It is easy to see that the graph $G=\left[F^{z}, H_{z}\right]$, where $F^{z}$ is $z-\mathrm{MNT}$, is MNT if $G+u v$ is traceable for $u \in F^{z}-z$ and $v \in H_{z}$.

Suppose $F$ is an $x y$-MNT graph and $F^{z}$ is a $z-\mathrm{MNT}$ graph, where $x$ and $z$ are universal vertices and $\left[F, H_{x}, H_{y}\right]$ and $\left[F^{z}, H_{z}\right]$ are MNT graphs of order $n \geq 10$. Then $\left\langle V(F) \cup V\left(H_{y}\right)\right\rangle-x$ and $F^{z}-z$ are themselves MNT. If each of $H_{x}, H_{y}$ and $H_{z}$ is either a $K_{1}$ or $K_{2}$, then by using Theorem 2.3 and results for the minimum size of MNT graphs of orders less than 10 given in [8] it is not difficult to check that $\left[F, H_{x}, H_{y}\right]$ and $\left[F^{z}, H_{z}\right]$ have size greater than $\left\lceil\frac{3 n}{2}\right\rceil$. Thus, since smallest MNT graphs of a specific order may arise from $x y$-MNT or $z$-MNT graphs, we searched, with the aid of a computer, for $x y-\mathrm{MNT}$ and $z$-MNT graphs of given order and smallest size in which neither $x$ nor $y$ nor $z$ is a universal vertex. The algorithm for $x y$-MNT graphs $F$ of order $n$ starts with an initial graph of order $n$ that is necessarily a subgraph of any $x y-\mathrm{MNT}$ graph of order $n$. From Lemmas 3.1 (a) and (b) we choose an $n$-path of the form $x y Q$ as the initial graph. The algorithm then performs a depth-first tree search, systematically adding all possible combinations of edges, but keeping the graph $x y$-nontraceable. When a predetermined upper bound on the number of edges is reached, that branch is terminated.

We could find all $x y$-MNT graphs of minimum size of order 5 up to 13 in reasonable computing time. These graphs are depicted in the Figures 36 . 

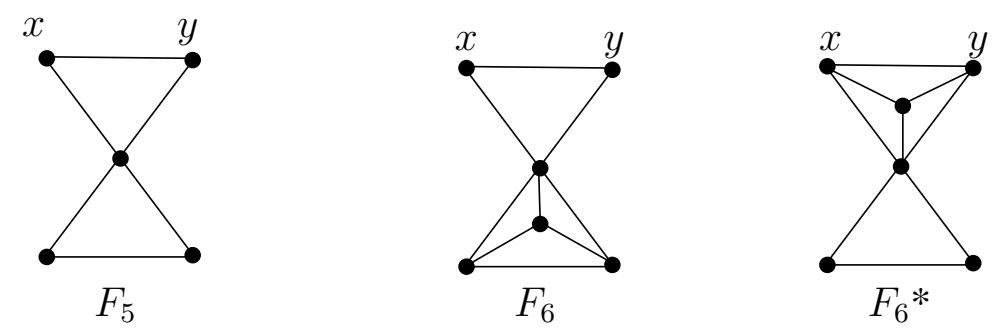

Fig. 3: Smallest $x y-M N T$ graphs of order 5 (6 edges) and order 6 (9 edges)
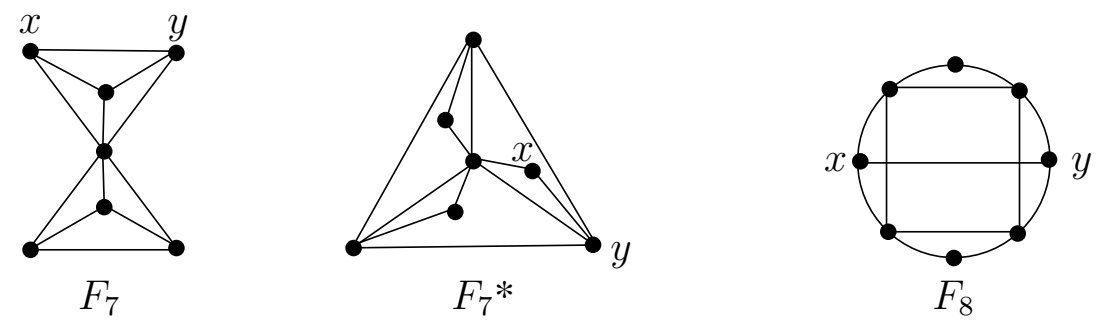

Fig. 4: Smallest $x y-M N T$ graphs of order 7 (12 edges) and of order 8 (13 edges)
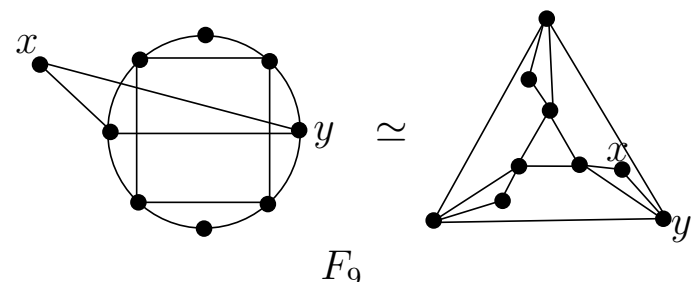

$F_{9}$

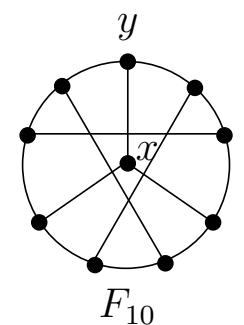

$F_{10}$

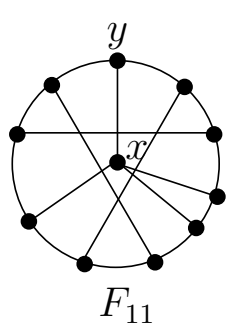

Fig. 5: Smallest $x y$-MNT graphs of order 9 (15 edges), order 10 (15 edges) and order 11 (17 edges) 

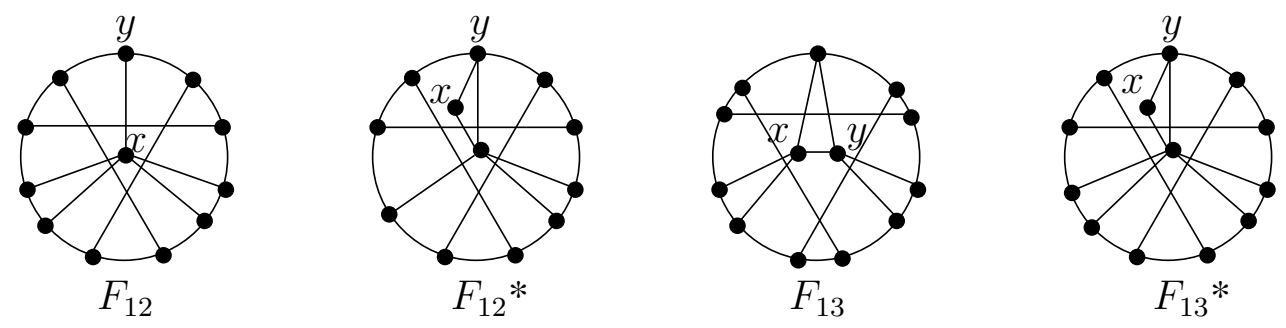

Fig. 6: Smallest $x y-M N T$ graphs of order 12 (19 edges) and order 13 (21 edges)

We also established, by computer, that the smallest $\mathrm{MNH}$ graphs $G_{14}$ of order 14 and size 22, and $G_{15}$ of order 15 and size 24 (see Figure 7) given in [9] are in fact $x y$-MNT.
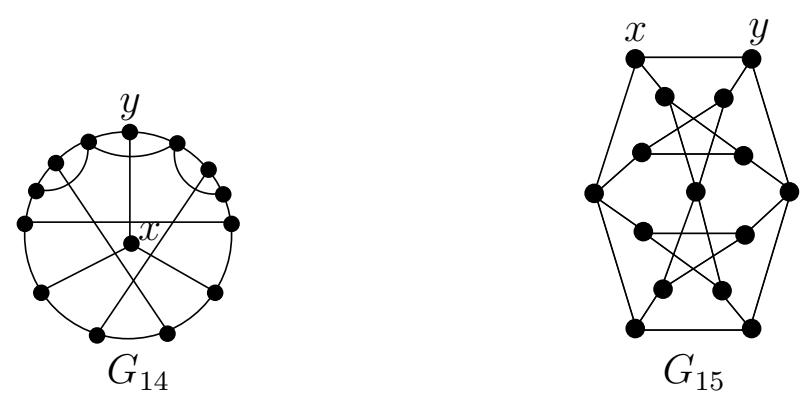

Fig. 7: Smallest maximal nonhamiltonian graphs $G_{14}$ and $G_{15}$

A similar search for $z$-MNT graphs of minimum size was undertaken for orders up to 12 . We noted that for $7 \leq n \leq 12$ all $z$-MNT graphs of minimum size can be constructed from $x y$-MNT graphs of minimum size in one or both of the following ways:

(1) Attach a $K_{1}$ to $x$ (or $y$ ) of a smallest $x y$-MNT graph of order $n-1$.

(2) Attach a $K_{2}$ to $x$ (or $y$ ) of a smallest $x y$-MNT graph of order $n-2$.

If $K_{i}, i=1,2$ is attached to $x$ (or $y$ ), then $z$ is $y$ (or $x$ ).

We therefore do not include sketches of these graphs. Table 1 gives the minimum sizes of $x y-\mathrm{MNT}$ graphs and $z-$ MNT graphs of orders up to 15 and 13 respectively.

\begin{tabular}{|l|c|c|c|c|c|c|c|c|c|c|c|}
\hline$n$ & 5 & 6 & 7 & 8 & 9 & 10 & 11 & 12 & 13 & 14 & 15 \\
\hline Smallest $x y$-MNT & 6 & 9 & 12 & 13 & 15 & 15 & 17 & 19 & 21 & $\leq 22$ & $\leq 24$ \\
\hline Smallest $z$-MNT & 5 & 7 & 9 & 12 & 14 & 16 & 16 & 18 & $\leq 20$ & & \\
\hline
\end{tabular}

Tab. 1: Sizes of smallest $x y-\mathrm{MNT}$ and $z-\mathrm{MNT}$ graphs 
Remark 3.5 We note the following:

(a) The xy-MNT graphs $F_{8}, F_{11}, F_{12}$ and $F_{13}$ are hamiltonian whereas the others in Figures 3 are MNH. The MNH ones for orders 5, 6, 7, 9 and 10 are those listed as the MNH graphs of smallest size in [9]. Each of the MNH graphs of orders 12 and 13 have one more edge than the corresponding MNH graphs of smallest size listed in [9]

(b) $N_{F_{j}}(x) \cap N_{F_{j}}(y) \neq \emptyset$ for $j \in\left\{5,6,6^{*}, 7,7^{*}, 9,12^{*}, 13,13^{*}\right\}$. In these cases it is easy to check that $\left[F_{j}, H_{x}, H_{y}\right]$ is $M N T$.

(c) The graph $F_{7}^{*}$ is also a $z-M N T$ graph (but not of smallest size), where $z$ is the universal vertex.

(d) The xy-MNT graphs $F_{5}, F_{6}, F_{8}$ and $F_{10}$ were used in [3] and [8] to construct smallest size MNT graphs of orders 7, 8, 9, 10, 12 and 13 .

(e) In $F_{10}$ and in $G_{15}, x$ and $y$ can be any two adjacent vertices of the graphs.

A cubic $x y$-MNT graph with $N(x) \cap N(y)=\emptyset$ of order $n$ can be used to construct an MNT graph of order $n^{\prime}=n+2$ or $n^{\prime}=n+3$ by attaching a $K_{1}$ to $x$ and a $K_{1}$ or $K_{2}$ to $y$. These MNT graphs realise the lower bound $\left\lceil\frac{3 n^{\prime}-2}{2}\right\rceil$. This is the construction used by Dudek, Katona and Wojda in [7] to produce MNT graphs of order $n$ and size $\left\lceil\frac{3 n-2}{2}\right\rceil$ for $n \geq 54$ as well as for $n \in$ $\{22,23,30,31,38,39,40,41,42,43,46,47,48,49,50,51\}$. This fact motivated us to search for cubic $x y$-MNT graphs of relevant orders. We used cubic graphs from Gordon Royle's Collection of small graphs (cf. [11]) and found $x y$-MNT graphs for orders 16, 18 and 22 (see Figure 8), but none of order 14. We also found $x y$-MNT graphs of orders 24, 26 and 30 (see Figure 9) from Brendan McKay's collection of cubic hypohamiltonian graphs (cf. [10]). We label these graphs $Q_{i}$, where $i$ is the order of the graph.

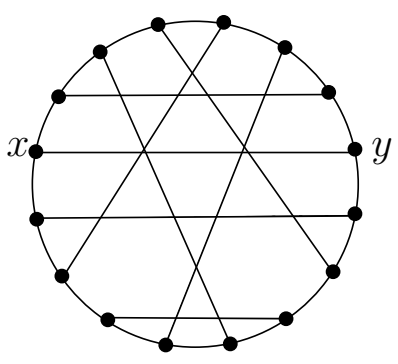

(a) Cubic graph $Q_{16}$

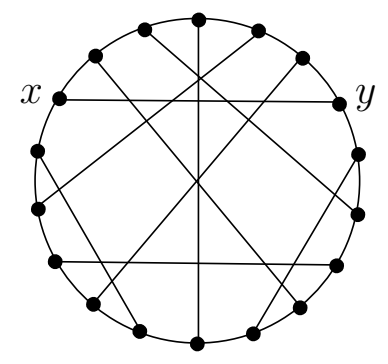

(b) Cubic graph $Q_{18}$

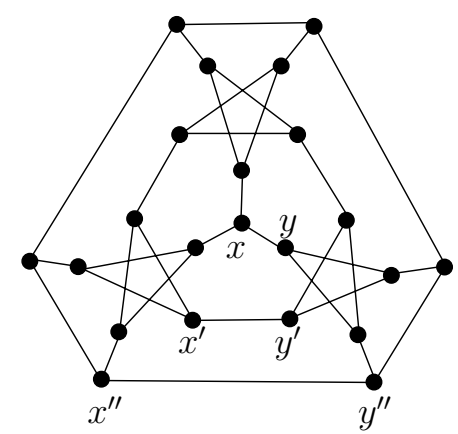

(c) Cubic graph $Q_{22}$

Fig. 8: Cubic $x y$-MNT graphs of order 16, 18 and 22 


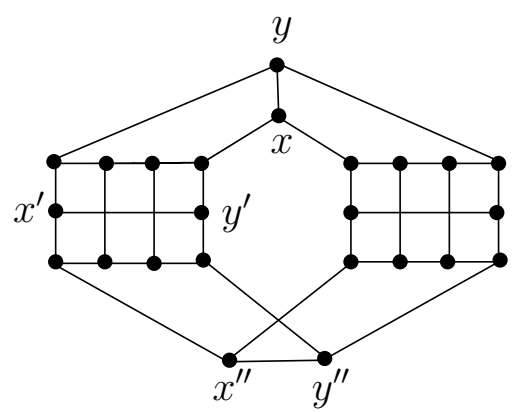

(a) Cubic graph $Q_{24}$

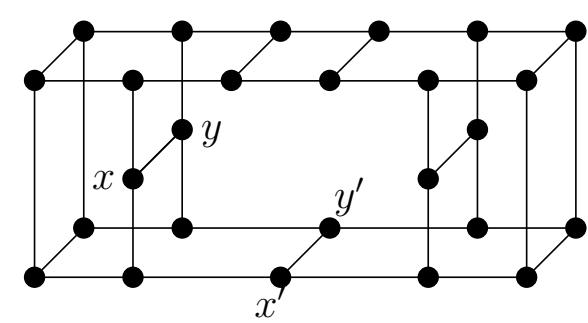

(b) Cubic graph $Q_{26}$

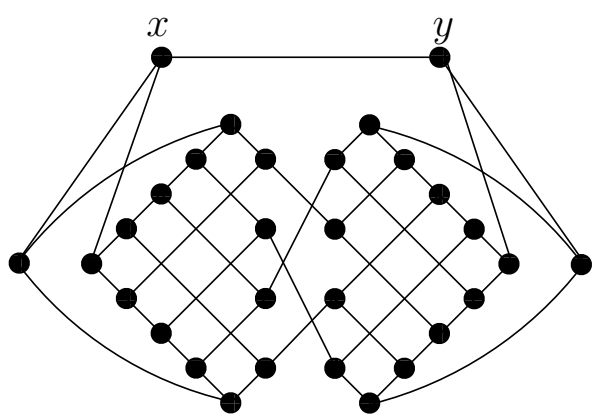

(c) Cubic graph $Q_{30}$

Fig. 9: Cubic hypohamiltonian $x y$-MNT graphs of order 24, 26 and 30

Remark 3.6 We note the following:

(a) The graphs $Q_{i}, i=16,18,22,24,26,30$ all have $N(x) \cap N(y)=\emptyset$ and neither $x$ nor $y$ is a universal vertex of $Q_{i}$.

(b) The graphs $Q_{16}, Q_{18}$ are hamiltonian. This is to be expected since the smallest MNH graphs of orders 16 and 18 listed in [9] have 25 and 28 edges respectively.

(c) In $Q_{30}, x$ and $y$ can be any two adjacent vertices of the graph and in $Q_{22}, Q_{24}$ and $Q_{26}$, where applicable, $x, y$ can be $x^{\prime}, y^{\prime}$ or $x^{\prime \prime}, y^{\prime \prime}$.

(d) There are no cubic $x y-M N T$ graphs of order 14.

\section{Main Results}

In this section we determine $g(n)$ for $n=10,11,14-21,24-29,32,33$.

Theorem 4.1 For $n \in\{18,19,20,21,24,25,26,27,28,29,32,33\}, g(n)=\left\lceil\frac{3 n-2}{2}\right\rceil$. 
Proof: Let $F=Q_{i}$ for $i=16,18,22,24,26,30, H_{x}=K_{1}$ and $H_{y}=K_{1}$ or $H_{y}=K_{2}$. The result then follows from Theorems 2.3 and 3.4 and Remark 3.6 (a).

We now prove that $g(n)=\left\lceil\frac{3 n}{2}\right\rceil$ for $n=10,11,14,15,16,17$.

We prove $g(n)=\left\lceil\frac{3 n}{2}\right\rceil$ for $n=10$ as a separate theorem using "analytic" arguments. Alternatively, this result can be obtained by using computer results. The cases $n=11,14,15,16$ and 17 are proved using computer results.

Theorem 4.2 The minimum size of an MNT graph of order 10 is 15 .

Proof: According to Theorem $2.3 g(10) \geq 14$. Suppose $G$ is an MNT graph of order 10 and size 14 . We define $M, S, m$ and $s$ as in Theorem 2.3. It follows from Theorem 2.2 that $s \neq 0$. We consider the various cases given in the proof of Theorem 2.3 .

Case 1 ( $\langle S\rangle$ has 3 components)

The smallest graph of order 10 for this case is the Zelinka type II graph depicted in Figure 10 which has 15 edges.

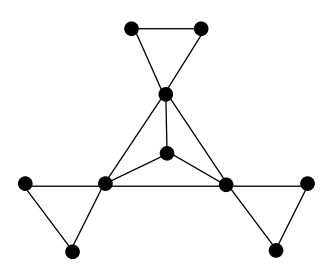

Fig. 10: Zelinka type II graph of order 10

Case $2\left(\langle S\rangle\right.$ has 2 components, $H_{1}$ and $\left.H_{2}\right)$

Case 2.1 $N_{G}\left(H_{1}\right)=N_{G}\left(H_{2}\right)$

According to Remark 2.4 (b) we need not consider this case.

Case 2.2 $N_{G}\left(H_{1}\right) \neq N_{G}\left(H_{2}\right)$

Case 2.2.1 $G-S$ is hamiltonian

From inequality (1) of Section 2 it follows that the only values of $m$ and $s$ that may produce an MNT graph $G$ of order 10 and 14 edges are $m=0$ and $s=2$, i. e. $G-S$ has no vertices of degree 2 . Hence $G-S$ is a cubic $x y-$ MNT graph of order 8 . We show that no such graph $G-S$ exists.

The subgraphs $G_{s}$ of $G$ can have one of the three structures depicted in Figure 11 


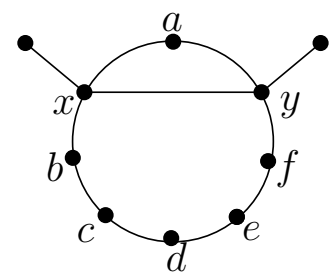

(a)

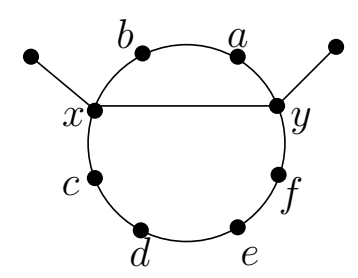

(b)

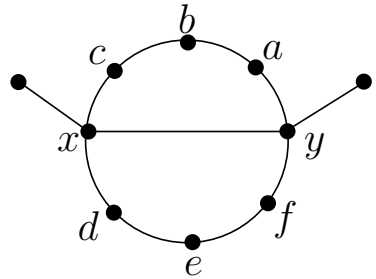

(c)

Fig. 11: Structures of $G_{s}$

We prove the subcase for $G_{s}$ shown in Figure 11 b). The proofs of the other subcases are similar.

Firstly $a c, b f \notin E(G)$, otherwise $G-S$ is $x y$-traceable. Due to symmetry all subcases follow from the following three cases.

(i) Suppose $a d \in E(G)$. Then $b c$, be $\notin E(G)$. Hence $G-S$ is not cubic.

(ii) Suppose $a e \in E(G)$. Then $b d \notin E(G)$. If $b c, d f \in E(G)$, then $G-S$ is $x y$-traceable.

(iii) Suppose $a f \in E(G)$. Then $b c, b e \notin E(G)$. If $b d, c e \in E(G)$, then $G-S$ is $x y$-traceable.

Thus there is no MNT graph $G$ with 14 edges having $G_{s}$ as subgraph.

\section{Case 2.2.2 $G-S$ is $\mathrm{MNH}$}

From inequality (2) of Section 2 it follows that the only value of $s$ that may produce an MNT graph $G$ of order 10 and 14 edges is $s=2$.

According to [9] the smallest MNH graph of order 8 has 15 edges and thus $e(G)>14$.

\section{Case $3(\langle S\rangle$ has 1 component, $H)$}

According to inequality (3) of Section 2 the only possibility for a graph of order 10 to have 14 edges is when $m=0$, i. e. when $d_{G}(v) \neq 2$ for all $v \in V(G)-S$.

For $s=1, e(G)=14 \Longleftrightarrow d_{G}(v)=3$ for all $v \in V(G)-S$.

For $s=2$, taking Lemma 2.1 into account, we have

$$
\begin{aligned}
e(G) & =\frac{1}{2}\left(\sum_{v \in S} d_{G}(v)+\sum_{v \in V(G)-S} d_{G}(v)\right) \\
& \geq \frac{1}{2}(4+4+6(3)+4)=15 .
\end{aligned}
$$

Thus we only consider $s=1$, i. e. $H \cong K_{1}$. Then, according to Lemma 2.7 $G-S$ is 2-connected.

Let $z$ denote the vertex adjacent to $H$ in $G$, and $F=G-S$. Then $d_{F}(z)=2$ and the other 8 vertices of $F$ must have degree 3 each. Note that $F$ is not traceable from $z$, otherwise $G$ will be traceable. We show that such a graph $G$ does not exist by attempting to construct $F$ by considering circumference cycles of $F$.

Let $C$ be a circumference cycle of $F$. We define, respectively, the degree deficiency, $d d(C)$, of $C$, and the degree deficiency, $d d(F-V(C))$, of $F-V(C)$ in $F$ as

$$
d d(C)=\sum_{v \in V(C)} d_{F}(v)-2|V(C)|
$$


and

$$
d d(F-V(C))=\sum_{v \in V(F-V(C))} d_{F}(v)-\sum_{v \in V(F-V(C))} d_{F-V(C)}(v) .
$$

Since $F$ is connected $d d(F-V(C))>0$ and thus

$$
d d(C) \geq d d(F-V(C))>0 .
$$

Since $F$ is not traceable from $z$ it follows that $c(F) \leq 8$. Suppose $C$ is a circumference cycle of $F$ of order $k \leq 8$. We denote the vertices on $C$ in an anti-clockwise direction by $x_{0}, x_{1}, \ldots, x_{k-1}$, where $x_{0}=z$ when $z \in V(C)$. We denote the vertices of $F-V(C)$ by $v_{0}, v_{1}, \ldots, v_{8-k}$, where $v_{0}=z$ when $z \in V(F-V(C))$.

Since similar techniques are used in proving the cases $c(F)=8$ and $c(F)=7$ as in the case $c(F)=6$, we only prove the following:

$c(F)=6$

(a) $z \in V(C)$

Now $d d(C)=5$ and the only graphs $\left\langle v_{0}, v_{1}, v_{2}\right\rangle$ with $0<d d\left(\left\langle v_{0}, v_{1}, v_{2}\right\rangle\right) \leq 5$ are $P_{3}$ and $K_{3}$. Each of $P_{3}$ and $K_{3}$ have vertices $v_{i}, v_{j} ; i \neq j ; i, j=0,1,2$ such that $d_{F-V(C)}\left(v_{i}\right)<3$ and $d_{F-V(C)}\left(v_{j}\right)<3$ and $v_{i}$ and $v_{j}$ are endvertices of a path of order 3. Since, according to Lemma 2.7, $F$ is 2-connected it follows that $v_{i}$ and $v_{j}$ have distinct neighbours on $C$ which contradicts Lemma 2.6. Hence $d_{F}\left(v_{i}\right)<3$ and $d_{F}\left(v_{j}\right)<3$, a contradiction.

(b) $z \in F-V(C)$

Now $d d(C)=6$ and the only graphs $\left\langle z, v_{1}, v_{2}\right\rangle$ with $0<d d\left(\left\langle z, v_{1}, v_{2}\right\rangle\right) \leq 6$ are $K_{1} \cup K_{2}, P_{3}$ and $K_{3}$. Using reasoning similar to that in (a) it can be seen that $\left\langle z, v_{1}, v_{2}\right\rangle$ cannot be isomorphic to $P_{3}$ or $K_{3}$. Now consider $K_{1} \cup K_{2}$. Let $V\left(K_{1}\right)=w_{1}$ and $V\left(K_{2}\right)=\left\{w_{2}, w_{3}\right\}$, where $z=w_{1}$ or $z=w_{2}$. Then $d_{F}\left(w_{3}\right)=3$. Then, according to Lemma 2.5 we may assume $w_{2} x_{0}, w_{3} x_{3} \in E(F)$, but then $w_{3} x_{i} \notin E(F)$ for $i=1,2,4,5$ and hence $d_{F}\left(w_{3}\right)<3$.

\section{$\boldsymbol{c}(\boldsymbol{F})$ at most 5}

Clearly at least one component of $F-V(C)$, say $A$, has at least two vertices. Then there are two distinct vertices in $A$ which, since $F$ is 2 -connected, have distinct neighbours on $C$. This contradicts Lemma 2.6 .

Thus $g(10) \geq 15$ and since there exist MNT graphs of order 10 and size 15 (for example, the graph in Figure 10 and $\left[F_{8}, K_{1}, K_{1}\right]$ which was first reported in [3]) it follows that $g(10)=15$.

In the proof of Theorem 4.5 we will consider degree sequences of graphs. Since all values in the degree sequences that we consider are less than 10, we will use, for example, the notation $123^{4} 4$ for a graph having degrees $1,2,3,3,3,3,4$. We use a computer search using the same algorithm as described in Section 3 but with a restriction on the degree sequence of a specific path, where we define the degree sequence of a path $P=v_{1} v_{2} \ldots v_{n}$ in $G$ as $d_{G}\left(v_{1}\right), d_{G}\left(v_{2}\right), \ldots, d_{G}\left(v_{n}\right)$. These extra restrictions enabled the relevant searches to be completed in a reasonable time. The following two lemmas describe the paths used for different cases in the computer algorithms.

Lemma 4.3 Suppose $F$ is an $x y-M N T$ graph with $n \geq 6$ and neither $x$ nor $y$ is a universal vertex of $F$. Then the following hold.

(a) There is a Hamilton path $x y P$ in $F$. 
(b) Let $u \in V(F)-\{x, y\}$. If $x \notin N(u)$, then $F$ has a Hamilton path $x y P u$. If $x \in N(u)$, then $F$ has a Hamilton path $x y P u Q$.

(c) If $w \in V(F)-\{x, y\}$ has degree 2 and $N(w)=\left\{v_{1}, v_{2}\right\}$ with $d\left(v_{i}\right)=4$ for $i=1$, 2 , then $F$ has a Hamilton path $x y P v_{i} w v_{j}$ or yxP $v_{i} w v_{j}$, where $\{i, j\}=\{1,2\}$.

\section{Proof:}

(a) and (b) follow directly from Lemma 3.1

(c) From Lemmas 3.2 (a) and (b) it follows that $v_{1}$ and $v_{2}$ are distinct from $x$ and $y$, and that not both are adjacent to $x$ or $y$. If $x$ and $v_{j}, j \in\{1,2\}$ are nonadjacent, then $F$ has a Hamilton path $x y P v_{i} w v_{j}$, where $\{i, j\}=\{1,2\}$. Similarly, if $y$ and $v_{j}, j \in\{1,2\}$ are nonadjacent, then $F$ has a Hamilton path $y x P v_{i} w v_{j}$, where $\{i, j\}=\{1,2\}$.

Lemma 4.4 Suppose $F$ is a $z-M N T$ graph with $n \geq 5$ and $z$ is not a universal vertex of $F$.

(a) There is a Hamilton path $P$ in $F-z$.

(b) Let $u \in V(F)-\{z\}$. If $z \notin N(u)$, then $F-z$ has a Hamilton path $u P$. If $z \in N(u)$, then $F-z$ has a Hamilton path PuQ.

(c) If $w \in V(F)-\{z\}$ has degree 2 and $N(w)=\left\{v_{1}, v_{2}\right\}$ with $d\left(v_{i}\right)=4$ for $i=1$, 2, then $F-z$ has a Hamilton path $P v_{i} w v_{j}$, where $\{i, j\}=\{1,2\}$.

\section{Proof:}

(a) and (b) are obvious.

(c) From Lemma 3.3 (b) $v_{1}$ and $v_{2}$ are not both adjacent to $z$. Say $v_{j}$ is not adjacent to $z$ for $j \in\{1,2\}$. Then $F+z v_{j}$ must be $z$-traceable. Therefore there is a Hamilton path $z v_{j} w v_{i} P$ in $F$. (Note the path cannot end in $w$, otherwise $F-z$ is hamiltonian, making $F z$-traceable.) Therefore $F-z$ has a Hamilton path $P v_{i} w v_{j}$, where $\{i, j\}=\{1,2\}$.

Theorem 4.5 For $n=11,14,15,16$ and $17, g(n)=\left\lceil\frac{3 n}{2}\right\rceil$.

Proof: According to Theorem 2.3, $g(n) \geq\left\lceil\frac{3 n-2}{2}\right\rceil$ for $n \geq 10$. In order to show that there are no MNT graphs of order $n$ and size $\left\lceil\frac{3 n-2}{2}\right\rceil$ for $n=11,14,15,16$ and 17, it follows from Theorem 2.2 and Remarks 2.4 (a) and (b), that we need only consider Cases 2.2 and 3 of Theorem 2.3. This implies from Remark 2.4(c) that we need only consider MNT graphs constructed from $x y-\mathrm{MNT}$ and $z-\mathrm{MNT}$ graphs. In some cases we are able to use Table 1 to determine the minimum size of an MNT graph of a specific order constructed from an $x y-\mathrm{MNT}$ graph and/or a $z-\mathrm{MNT}$ graph of minimum size. If not, we consider values of $m$ and $s$ that could possibly produce MNT graphs $G$ of order $n$ with $\left\lceil\frac{3 n-2}{2}\right\rceil$ edges 
and determine possible degree sequences for such graphs. In most cases, for the sake of brevity, we will only state the degree sequence(s) of $G$ and the relevant lemma(s) used for the computation. When determining the degree sequences for $G$, we use Lemmas 2.1, 3.2 a) and 3.3 (a), and the fact that $d(v) \geq 3$ for $v \in V(G)-S-M$. In the case where $G-S$ is a hamiltonian $x y$-MNT graph we use the fact that $d_{G-S}(x) \geq 3$ and $d_{G-S}(y) \geq 3$ (see Case 2.2.1 of Theorem 2.3). For all cases, no graphs with the relevant degree sequences were found when doing a computer search and hence we do not state this in every case.

Case I: $n=11$

According to Table 1 the smallest MNT graphs of order 11 which can be constructed from $x y$-MNT or $z-$ MNT graphs have 17 edges. Hence $g(11)=17$. The graphs $\left[F_{8}, K_{1}, K_{2}\right]$ and $\left[F_{9}, K_{1}, K_{1}\right]$ are examples of such graphs.

Case II: $n=14$

From Table 1 it follows that the smallest MNT graph of order 14 that can be constructed from an $x y-\mathrm{MNT}$ graph has 21 edges.

Suppose $G=\left[F, H_{z}\right]$ is an MNT graph of order 14 and size 20. Then from inequality (3) of Section 2 we can only have $m=0$. We consider subcases according to possible values of $s$.

(i) $m=0$ and $s=1$ : The only possible degree sequence is $13^{13}$ and we use Lemma 4.4 (a) in our computation.

(ii) $m=0$ and $s=2$ : It follows from Table 1 that the minimum number of edges is 21 .

We have shown that $g(14)=21$. The graphs $\left[F_{12}, K_{1}, K_{1}\right],\left[F_{12}^{*}, K_{1}, K_{1}\right],\left[F_{11}, K_{1}, K_{2}\right]$ and $\left[F_{10}, K_{2}, K_{2}\right]$ are examples of MNT graphs of order 14 and size 21 .

Case III: $n=15$

From Table 1 it follows that the smallest MNT graph of order 15 that can be constructed from an $x y$-MNT graph has 23 edges.

Suppose $G=\left[F, H_{z}\right]$ is an MNT graph of order 15 and size 22, where $F$ is a $z$-MNT graph. Then from inequality (3) of Section 2 we have $m \leq 1$. Table 2 summarizes the possible subcases.

\begin{tabular}{|llll|}
\hline$m$ & $s$ & degree sequence & Lemma used for computer search \\
\hline 0 & 1 & $13^{13} 4$ & $4.4(\mathrm{~b})$ if $d_{G}(z)=3$ with $d(u)=4 ; 4.4$ (a) if $d_{G}(z)=4$ \\
0 & 2 & $223^{12} 4$ & 4.4 (a) [Same as Case II(i)] \\
1 & 1 & $123^{11} 44$ & 4.4 (c) \\
1 & 2 & None & \\
\hline
\end{tabular}

Tab. 2: Subcases for $n=15$, where $G-S$ is $z-\mathrm{MNT}$

We have thus shown that $g(15)=23$. The graphs [ $\left.F_{13}, K_{1}, K_{1}\right]$, [ $\left.F_{13} *, K_{1}, K_{1}\right],\left[F_{12}, K_{1}, K_{2}\right]$, $\left[F_{12} *, K_{1}, K_{2}\right]$ and $\left[F_{11}, K_{2}, K_{2}\right]$ are examples of MNT graphs of order 15 and size 23.

Case IV: $n=16$

We show that no MNT graph $G$ of order 16 and size 23 exists.

We first consider the case where $G-S$ is an $x y-$ MNT graph and $G-S$ is MNH. The smallest such MNT graph of order 16 has $G_{14}$ as subgraph and has 24 edges. We now assume $G-S$ is hamiltonian. It follows from inequality (1) of Section 2 that $m+s \leq 2$. Thus $m=0$ and $s=2$, and the only possible degree sequence is $113^{12} 44$ since $d_{G-S}(x) \geq 3$ and $d_{G-S}(y) \geq 3$. Hence $G-S$ must be cubic. Applying Lemma 4.3 a) and a computer search, no graph was found. 
We next consider the case where $G-S$ is a $z-M N T$ graph. If such a graph exists then from inequality (3) of Section 2 we have $m=0$. The possible subcases are:

(i) $m=0$ and $s=1$ : The degree sequence is $13^{15}$ and we use Lemma 4.4 (a).

(ii) $m=0$ and $s=2$ : No degree sequence for $G$ is possible.

We have thus shown that $g(16)=24$ and that $\left[G_{14}, K_{1}, K_{1}\right]$ is an MNT graph with order 16 and size 24 (see Figure 7).

Case V: $n=17$

We show that no MNT graph $G$ of order 17 and size 25 exists. We first consider the case where $G-S$ is an $x y$-MNT graph and $G-S$ is MNH. The smallest such MNT graphs of order 17 have either $G_{14}$ or $G_{15}$ as subgraphs and have 26 edges. We thus need to consider the case where $G-S$ is a hamiltonian $x y$-MNT graph in which case $m+s \leq 3$ (inequality (1)); and the case where $G-S$ is a $z-$ MNT graph in which case $m \leq 1$ (inequality (3)). Table 3 summarizes all possible subcases.

\begin{tabular}{|c|c|c|c|c|}
\hline$G-S$ & $m$ & $s$ & degree sequence & Lemma used for computation \\
\hline hamiltonian & 0 & 2 & $113^{13} 45$ or & 4.3 a) \\
\hline \multirow{3}{*}{$x y-\mathrm{MNT}$} & 0 & 2 & $113^{12} 444$ & 4.3 b) with $d(u)=4$ \\
\hline & 0 & 3 & $1223^{12} 45$ & 4.3 a) \\
\hline & 1 & 2 & $1123^{10} 4444$ & $4.3(\mathrm{c})$ \\
\hline \multirow[t]{4}{*}{$z-\mathrm{MNT}$} & 0 & 1 & $13^{15} 4$ & $\begin{array}{l}4.4\left(\text { b) if } d_{G}(z)=3 \text { with } d(u)=4 ;\right. \\
4.4 \text { a) if } d_{G}(z)=4\end{array}$ \\
\hline & 0 & 2 & $223^{14} 4$ & 4.4 a) [Same as Case IV(i)] \\
\hline & 1 & 1 & $123^{13} 44$ & $4.4(\mathrm{c})$ \\
\hline & 1 & 2 & None & \\
\hline
\end{tabular}

Tab. 3: Subcases for $n=17$, where $G-S$ is hamiltonian $x y-\mathrm{MNT}$ or $z-\mathrm{MNT}$

Hence $g(17)=26$ and $\left[G_{14}, K_{1}, K_{2}\right]$ and $\left[G_{15}, K_{1}, K_{1}\right]$ are examples of MNT graphs of order 17 and size 26 .

Obviously, there can be some doubt about the correctness of an algorithm when no graphs are found. The fact that MNH graphs of certain orders that were found in [9] were also found with this algorithm is reassuring. Furthermore, since the algorithm does not take into account isomorphisms of graphs, it is unlikely that a graph would be missed since the algorithm has usually more than one chance to find a graph if it exists. The source code for the algorithm can be viewed at [4].

We summarize the results contained in [8] and in Theorems 4.1, 4.2 and 4.5 in the following theorem:

Theorem 4.6 Let $g(n)$ denote the minimum number of edges of an MNT graph of order $n$. Then

$$
g(n)= \begin{cases}\left\lceil\frac{3 n}{2}\right\rceil & \text { for } n \in J=\{10,11,14,15,16,17\} \\ \left\lceil\frac{3 n-2}{2}\right\rceil & \text { for } n \geq 10, n \notin J \cup K \text { where } K=\{34,35,36,37,44,45,52,53\} .\end{cases}
$$




\section{Conclusion}

The smallest size of an MNT graph of order $n$ has now been determined for all except 8 values of $n$, namely $n \in K=\{34,35,36,37,44,45,52,53\}$. The minimum size of MNT graphs of order $n \geq 18$, where $n \notin K$, is $\left\lceil\frac{3 n-2}{2}\right\rceil$ and graphs of this size are constructed from cubic $x y$-MNT graphs. We suspect that $g(n)=\left\lceil\frac{3 n-2}{2}\right\rceil^{2}$ also for $n \in K$ and that these remaining cases could be resolved by finding cubic $x y$-MNT graphs of orders 32, 34, 42 and 50 either by construction or by the use of more sophisticated algorithms.

\section{Acknowledgements}

We wish to express our thanks to Dr Marietjie Frick who initiated this work and for her helpful comments on our draft manuscript.

\section{References}

[1] B. Bollobás. Extremal graph theory. London: Academic Press, 1978.

[2] J. Bondy. Variations on the hamiltonian theme. Canad. Math. Bull., 15:57-62, 1972.

[3] F. Bullock, M. Frick, J. Singleton, S. van Aardt, and C. Mynhardt. Maximal nontraceable graphs with toughness less than one. Electronic Journal of Combinatorics, 15 R18, 2008.

[4] A. Burger. Maximal nontraceable graphs, February 2013. URL http://www.vuuren.co.za-> Repositories

[5] L. Clark and R. Entringer. Smallest maximally nonhamiltonian graphs. Period. Math. Hung., 14:57-68, 1983.

[6] L. Clark, R. Entringer, and H. Shapiro. Smallest maximally nonhamiltonian graphs ii. Graphs and Combin., 8: 225-231, 1992.

[7] A. Dudek, G. Katona, and A. Wojda. Hamiltonian path saturated graphs with small size. Discrete App. Math., 154(9):1372-1379, 2006.

[8] M. Frick and J. Singleton. Lower bound for the size of maximal nontraceable graphs. Electronic Journal of Combinatorics, 12(1) R32, 2005.

[9] X. Lin, W. Jiang, C. Zhang, and Y. Yang. On smallest maximally nonhamiltonian graphs. Ars Combin., 45: 263-270, 1997.

[10] B. McKay. Combinatorial data page, February 2013. URL http://cs.anu.edu.au/ bdm/data/ graphs.html.

[11] G. Royle. Gordon Royle's Small graphs, February 2013. URL http://school •maths . uwa . edu . au/ qordon/remote/cubics/

[12] Z. Skupień. Hamiltonian circuits and path coverings of vertices in graphs. Colloq. Math., 30:295-316, 1974.

[13] Z. Skupień. Hamiltonian shortage, path partitions of vertices, and matchings in a graph. Colloq. Math., 36 : 305-318, 1976.

[14] B. Zelinka. Graphs maximal with respect to absence of hamiltonian paths. Discussiones Mathematicae. Graph Theory, 18:205-208, 1998. 